\title{
Explicit solutions of supersymmetric KP hierarchies: Supersolitons and solitinos
}

\author{
A. Ibort, L. Martínez Alonso, and E. Medina Reus \\ Departamento de Física Teórica, Universidad Complutense, E-28040 Madrid, Spain
}

(Received 13 December 1995; accepted for publication 8 July 1996)

Wide classes of explicit solutions of the Manin-Radul and Jacobian supersymmetric KP hierarchies are constructed by using line bundles over complex supercurves based on the Riemann sphere. Their construction extends several ideas of the standard KP theory, such as wave functions, $\bar{\partial}$-equations and $\tau$-functions. Thus, supersymmetric generalizations of $N$-soliton solutions, including a new purely odd "solitino" solution, as well as rational solutions, are found and characterized.

(C) 1996 American Institute of Physics. [S0022-2488(96)02112-3]

\section{INTRODUCTION}

The most appealing aspects of the theory of the KP hierarchy have a rich geometrical content and it is likely that this fact is at the heart of the relevance of integrable systems in modern theoretical physics. ${ }^{1,2}$ For example, a common feature of the explicit methods of solutions is that they frequently involve geometric objects such as Grassmannians, algebraic curves, Abelian functions, and holomorphic line bundles. ${ }^{3-9}$ This also happens to be true in the context of supersymmetric KP (SKP) hierarchies ${ }^{10,11}$ and it is expected that some of these integrable systems are to find applications in string theory and quantum gravity. ${ }^{12-14}$ The aim of this article is to show how line bundles over complex supercurves can be used to provide efficient methods of solution for the SKP hierarchies formulated by Manin and Radul (MRKP) ${ }^{15}$ and Mulase and Rabin (JKP). ${ }^{10,11}$ Both hierarchies have attracted much attention because of their important mathematical content. Apart from sharing many of the standard integrability properties, among their reductions they have a supersymmetric generalization of the KdV hierarchy with a rich Hamiltonian structure ${ }^{15-17}$ and they are relevant from the point of view of superconformal algebra. ${ }^{18-20}$ A basic goal of our study is to generalize some of the standard methods of solution arising in the KP theory and to characterize the classes of solutions they determine. In particular we want to investigate solutions of soliton type and their relationship with the standard KP solitons. To this end we extend some strategies of the Grassmannian approach for constructing several types of solutions and apply them to the SKP hierarchies.

This work is based on the concept of super-wave function for a system of Sato equations of supersymmetric type. As it is known from our experience with the KP hierarchy, the notion of wave function is what allows us to derive methods of solution based on the resolution of analytic problems. Despite the seemingly great diversity of these methods, they exhibit a common ingredient: the presence of holomorphic line bundles ${ }^{6-8}$ whose sections turn to be the wave functions. Actually, the wave functions are special types of sections satisfying certain conditions as functions of the spectral parameter. These conditions are formulated in several forms depending on the particular approach which is being adopted. For example, they may be expressed by means of a $\bar{\partial}$-equation ${ }^{21,22}$ or by selecting a subspace of an infinite-dimensional Grassmanian. ${ }^{7}$ In this article we characterize the super-wave functions as certain sections of deformations of holomorphic line bundles over complex supercurves based on the Riemann sphere together with the corresponding points in the super-Grassmannian. Finally we also offer a $\bar{\partial}$-equation description of them. Then we compute several families of solutions including supersymmetric $N$-soliton solutions possessing an interesting composite odd structure, and a genuine supersymmetric family of solutions without a bosonic counterpart, unrelated to any class of solution of the standard KP theory, that we call solitino solutions. They exhibit a remarkable nonlinear superposition principle and a Fourier-like 
dependence on the bosonic variables. It is worth mentioning that the solutions found in the present work can also be determined, although in a less straigthforward way, through the bilinear formalism proposed in Ref. 23.

The first part of Section II is concerned with a brief review of SKP hierarchies in terms of pseudodifferential operators, including the formulation of Sato equations. The notion of superwave function is analyzed in the second part of this section. In particular, we describe in detail the derivation of the corresponding expressions for the vacuum super-wave functions. The characterization of super-wave functions as sections of (super) holomorphic line bundles over complex supermanifolds is described in the first part of Section III, and finally in Section IV we relate the above constructions to the supergroup and bilinear formalism.

\section{SUPER KP HIERARCHIES}

\section{A. Sato equations for super KP hierarchies}

The MRKP and JKP hierarchies can be described in a unified way with the help of an algebra $\mathscr{E}$ of super-pseudodifferential operators: ${ }^{24}$

$$
\left.X=\sum_{n \leqslant N} a_{n}(x, \theta, \mathbf{t}, \boldsymbol{\tau}) D^{n},\right\urcorner \quad N \in \mathbb{N},
$$

where the coefficients $a_{n}$ are elements of a supercommutative algebra

$$
\mathscr{S}=\mathbb{C}[[x, \mathbf{t}]] \otimes \Lambda(\theta, \boldsymbol{\tau}) \otimes \mathscr{C} .
$$

Here, $x$ is an even variable, $\theta$ is an odd variable, $\mathbf{t}=\left\{t_{n}\right\}_{n=1}^{\infty}$ and $\boldsymbol{\tau}=\left\{\tau_{n}\right\}_{n=1}^{\infty}$ are infinite sets of even and odd variables, respectively, and $\mathscr{A}$ denotes a given complex Grassmann algebra of finite or infinite dimension. The superdifferential operator $D$ is defined as the derivation on $\mathscr{S}$ given by

$$
D=\frac{\partial}{\partial \theta}+\theta \frac{\partial}{\partial x},
$$

satisfying the identity

$$
D^{2}=\partial \equiv \frac{\partial}{\partial x} .
$$

We will denote by $X=X_{+}+X_{-}$the decomposition of elements $X \in \mathscr{E}$ into positive ( $n \geqslant 0$ ) and negative $(n<0)$ powers $D^{n}$ of $D$.

Both algebras $\mathscr{S}$ and $\mathscr{E}$ have natural $Z_{2}$-graduations

$$
\left.\mathscr{S}=\mathscr{S}_{\underline{0}} \oplus \mathscr{S}_{\underline{1}},\right\urcorner \quad \mathscr{E}=\mathscr{E}_{0} \oplus \mathscr{E}_{1} .
$$

An operator (1) is said to have parity $p$ if and only if $(\forall n \leqslant N)$ the coefficients $a_{n}$ have parity $n+p \bmod 2$. We may also introduce body maps $\epsilon$ on $\mathscr{S}$ and $\mathscr{E}$ (Ref. 24) and, in particular, the body of an operator (1) is given by

$$
\epsilon(X)=\sum_{n \text { even }} \epsilon\left(a_{n}\right) D^{n} .
$$

Notice that $\epsilon(X)$ is not the even part of $X$. 
The Sato equations for the SKP hierarchies can be written as

$$
\begin{gathered}
\frac{\partial K}{\partial t_{n}}=-\left(K \partial^{n} K^{-1}\right)_{-} K, \\
\frac{D K}{D \tau_{n}}=-\left(K A_{n} K^{-1}\right)_{-} K, \neg n \geqslant 1,
\end{gathered}
$$

for an even $K \in \mathscr{E}$ of the form

$$
\left.K=1+\sum_{n=1}^{\infty} w_{n}(x, \theta, \mathbf{t}, \boldsymbol{\tau}) D^{-n} \cdot\right\urcorner
$$

Notice that $p\left(w_{2 n}\right)=0$ and $p\left(w_{2 n+1}\right)=1$ so that $p(K)=0$.

For the MRKP hierarchy the superderivations $D / D \tau_{n}$ and the operators $A_{n}$ in (2) are

$$
\frac{D}{D \tau_{n}}=\frac{\partial}{\partial \tau_{n}}-\sum_{m \geqslant 1} \tau_{m} \frac{\partial}{\partial t_{n+m-1}}, \neg A_{n}=D^{2 n-1} .
$$

while for the JKP version

$$
\frac{D}{D \tau_{n}}=\frac{\partial}{\partial \tau_{n}}, \neg A_{n}=\left(D-\theta D^{2}\right) D^{2 n-2}=\frac{\partial}{\partial \theta} D^{2 n-2}
$$

These hierarchies generalize the standard KP hierarchy in the sense that, as one easily shows, the body $\epsilon(K)$ of a solution $K$ of (2) satisfies the Sato equations for the KP hierarchy in the variables $t_{n}$.

By identifying the coefficients of the powers of $D$ in the system (2) one finds infinite systems of superdifferential equations involving the superfunctions $\left\{w_{n}\right\}$. Simpler equations can be obtained by imposing a reduction condition of the type ${ }^{15}$

$$
\frac{\partial K}{\partial t_{r}}=0, \neg
$$

for some $r \geqslant 2$. Indeed, if one introduces the operator

$$
L=K D K^{-1}=D+\sum_{n=1}^{\infty} u_{n} D^{-n+1}, \neg
$$

the constraint (4) is equivalent to

$$
\left(L^{2 r}\right)_{-}=\left(K D^{2 r} K^{-1}\right)_{-}=0,
$$

and it turns out that the operator

$$
\mathscr{B}=L^{2 r}
$$

satisfies the Lax equations

$$
\frac{\partial \mathscr{B}}{\partial t_{n}}=\left[\left(\mathscr{B}^{n / r}\right)_{+}, \mathscr{B}\right] \cdot \neg
$$


The coefficients of the expansion of $\left(\mathscr{b}^{n / r}\right)_{+}$in powers of $D$ are differential polynomials in the coefficients of $\mathscr{L}$ (Ref. 15), so that (6) leads to a system of superdifferential equations for the coefficients of $\mathscr{B}$ which constitutes a supersymmetric generalization of the $r$ th KdV hierarchy.

For example if we asssume (4) for $r=2$, then the operator $\mathscr{B}=L^{4}$ is of the form

$$
\mathscr{b}=D^{4}+v_{1} D+v_{0},
$$

where $v_{0}$ and $v_{1}$ can be expressed in terms of the coefficients of $K$ as

$$
\left.v_{0}=-2\left[\partial w_{2}+\left(\partial w_{1}\right) w_{1}\right], \neg v_{1}=-2 \partial w_{1} .\right\urcorner
$$

Furthermore, it is easy to find

$$
\left(\mathscr{L}^{3 / 2}\right)_{+}=D^{6}+\frac{3}{2} v_{1} D^{3}+\frac{3}{2} v_{0} D^{2}+\frac{3}{4} \partial v_{1} D+\frac{3}{4} \partial v_{0},
$$

so that the equation corresponding to (6) for $n=3$ reads $\left(t=t_{3}\right)$

$$
\begin{gathered}
\frac{\partial v_{0}}{\partial t}=\frac{1}{4}\left[\partial\left(\partial^{2} v_{0}+3 v_{1} D v_{0}+3 v_{0}^{2}\right)\right], \\
\frac{\partial v_{1}}{\partial t}=\frac{1}{4}\left[\partial\left(\partial^{2} v_{1}+3 v_{1} D v_{1}+6 v_{0} v_{1}\right)\right],
\end{gathered}
$$

which is the supersymmetric Korteweg-de Vries (SKdV) equation of Manin-Radul. ${ }^{15}$

\section{B. Super-wave functions for super KP hierarchies}

The notion of super-wave function for a SKP hierarchy requires the introduction of a spectral parameter $(z, \alpha)$, where $z$ denotes a complex variable and $\alpha$ is a new Grassmann variable. The superparameter $(z, \alpha)$ represents local supercoordinates on a complex supercurve $\mathscr{C}$ (see for instance, Ref. 25, and references therein for the notions of supergeometry used in the following) with structure sheaf $\mathscr{O}$ and not being necessarily a super-Riemann surface (SRS). Let $p$ be a point in the body of $\mathscr{C}$ and $D$ a disk containing $p$. We shall denote by $\mathscr{O}^{*}$ the restriction of sheaf of superfunctions $\mathscr{O}$ to $D-\{p\}$.

Definition $1:{ }^{11-20,24}$ A vacuum super-wave function is a superfunction $f_{0} \in \mathscr{O} * \otimes \mathscr{S}$ that satisfies

$$
\begin{gathered}
\frac{\partial f_{0}}{\partial t_{n}}=\partial^{n} f_{0}, \\
\frac{D f_{0}}{D \tau_{n}}=A_{n} f_{0}, \neg n \geqslant 1 .
\end{gathered}
$$

Hence, a super-wave function $f_{0}$ depends on the variables $(z, \alpha ; x, \theta, \mathbf{t}, \boldsymbol{\tau})$, and is a solution of the linear system (9). We can find solutions of (9) using the ansazt,

$$
\left.f_{0}=V e^{z x+\alpha \theta},\right\urcorner
$$

with $V$ a super-pseudodifferential operator in $\mathscr{E}$ and satisfying the initial condition $\left.V\right|_{\mathbf{t}=\mathbf{0}, \tau=\mathbf{0}}=1$. Then, $V$ must verify

$$
\frac{\partial V}{\partial t_{n}}=\partial^{n} \cdot V
$$




$$
\left.\frac{D V}{D \tau_{n}}=A_{n} \cdot V,\right\urcorner n \geqslant 1 .
$$

It is easy to see that for the MRKP hierarchy

$$
V=\exp \left[\sum_{n=1}^{\infty} t_{n} \partial^{n}\right] \cdot\left[1+\sum_{n=1}^{\infty} \tau_{n} D^{2 n-1}\right]
$$

and

$$
f_{0}=\exp \left[z x+\alpha \theta+\sum_{n=1}^{\infty} z^{n} t_{n}+(\alpha-z \theta) \sum_{n=1}^{\infty} z^{n-1} \tau_{n}\right] .
$$

Respectively for the JKP hierarchy

$$
V=\exp \left[\sum_{n=1}^{\infty} t_{n} \partial^{n}+\sum_{n=1}^{\infty} \tau_{n} \frac{\partial}{\partial \theta} D^{2 n-2}\right]
$$

and

$$
f_{0}=\exp \left[z x+\alpha \theta+\sum_{n=1}^{\infty} z^{n} t_{n}+\alpha \sum_{n=1}^{\infty} z^{n-1} \tau_{n}\right] .
$$

Henceforth we will write both expressions of the vacuum super-wave function $f_{0}$ as

$$
f_{0}=\exp \left[z x+\sum_{n=1}^{\infty} z^{n}\left(t_{n}+\theta \tau_{n}\right)+(\alpha-\lambda)\left(\theta+\sum_{n=1}^{\infty} z^{n-1} \tau_{n}\right)\right], \neg
$$

where $\lambda=\lambda(z, \theta, \tau)$ denotes a function which for the MRKP hierarchy is

$$
\left.\lambda(z, \theta, \boldsymbol{\tau})=z \theta-\sum_{n=1}^{\infty} z^{n} \tau_{n},\right\urcorner
$$

and for the JKP hierarchy is given by

$$
\lambda(z, \theta, \boldsymbol{\tau})=z \theta
$$

For brevity's sake, we will often omit the arguments of the superfunctions which are irrelevant or clear from the context.

It should be noticed that

$$
D f_{0}=(\lambda-\alpha) f_{0}, \neg D^{2} f_{0}=z f_{0}, \neg
$$

so that the action of inverse powers of $D$ on $f_{0}$ may be defined as

$$
\left.D^{-1} f_{0}=D \cdot D^{-2} f_{0}=(\lambda-\alpha) z^{-1} f_{0} \cdot\right\urcorner
$$

It is now evident that we can "dress" the vacuum super-wave function to obtain solutions of Sato's equations (2) introducing the notion of a super-wave function.

Let $H(\Omega)$ denote the set of complex valued holomorphic functions of $z$ on a certain region $\Omega$ of the complex plane, and $\sigma_{\Omega}=H(\Omega) \otimes \Lambda(\alpha)$ the superalgebra of superfunctions of the trivial (1|1)-superspace over $\Omega$. 
Definition 2: Let $\Omega$ and $\mathcal{O}_{\Omega}$ as before. A super-wave function for a SKP hierarchy is an even element $f=f(z, \alpha ; x, \theta ; \mathbf{t}, \boldsymbol{\tau})$ of the algebra

$$
\mathscr{O}_{\Omega} \otimes \mathrm{C}[[x, \mathbf{t}]] \otimes \Lambda(\theta, \boldsymbol{\tau}) \otimes \mathscr{A},
$$

which satisfies the properties:

(P1) $f$ is a solution of an infinite system of equations:

$$
\begin{gathered}
\frac{\partial f}{\partial t_{n}}=P_{n} f, \\
\frac{D f}{D \tau_{n}}=Q_{n} f, \neg n \geqslant 1,
\end{gathered}
$$

with $P_{n}, Q_{n} \in \mathscr{E}$ being superdifferential operators

$$
\left(P_{n}\right)_{-}=\left(Q_{n}\right)_{-}=0 .
$$

(P2) $f$ can be decomposed as a product:

$$
\left.f=f^{\prime} \cdot f_{0},\right\urcorner
$$

where $f^{\prime}$ admits a Laurent expansion on $r<|z|<r^{\prime}$ of the form:

$$
\left.f^{\prime}=1+\sum_{n=1}^{\infty} w_{2 n} z^{-n}+(\alpha-\lambda) \sum_{n=1}^{\infty} w_{2 n-1} z^{-n},\right\urcorner
$$

with $w_{n} \in \mathscr{S}$ for all $n \geqslant 1$.

Then a super-wave function $f$ is obtained by dressing the vacuum super-wave function $f_{0}$ using an operator $K$ satisfying Sato's equations.

Theorem 1: Let $f$ be a super-wave function for an SKP hierarchy and $f_{0}$ the vacuum superwave function (11), then there is an operator $K$ such that

$$
\left.f=K f_{0} \cdot\right\urcorner
$$

and $K$ verifies Sato's equations (2). Conversely, any solution of Sato's equations (2) provides a super-wave function $f$ for a SKP hierarchy by means of Eq. (19).

Proof: To prove this fact, we observe that as a consequence of properties (14) and (15) of $f_{0}$, the operator $K$ in (19) has the required form (3) with the coefficients $w_{n}$ being the same as those of the expansion (18) of $f^{\prime}$. On the other hand (16), (19) and (9) imply

$$
\frac{\partial K}{\partial t_{n}} f_{0}+K \partial^{n} f_{0}=P_{n} K f_{0},
$$

so that we deduce

$$
\frac{\partial K}{\partial t_{n}} K^{-1}=P_{n}-K \partial^{n} K^{-1}
$$

But this equation obviously leads to

$$
P_{n}=\left(K \partial^{n} K^{-1}\right)_{+}
$$

and 


$$
\frac{\partial K}{\partial t_{n}}=-\left(K \partial^{n} K^{-1}\right)_{-} K
$$

In the same way one shows that $K$ satisfies the Sato equations for the flows associated with the variables $\tau_{n}$. Thus, we have obtained in this way a one-to-one correspondence between solutions $K$ of Sato's equation and super-wave functions $f_{0}$.

Conversely, if $f=K f_{0}$, then because of (2), and (9), $f$ verifies (16) with $P_{n}=\left(K \partial^{n} K^{-1}\right)_{+}$and $Q_{n}=\left(K A_{n} K^{-1}\right)_{+}$. Moreover, because of the expansion (3) of the operator $K$ follows the expansion (18) of the transition superfunction $f^{\prime}$.

It is worth noticing that, as it should be expected, the body of a super-wave function for a SKP hierarchy constitutes a wave function for the standard KP hierarchy.

\section{LINE BUNDLES AND EXPLICIT SOLUTIONS OF THE SKP HIERARCHIES}

\section{A. Super-wave functions as sections of line bundles}

Contrary to the situation in the bosonic case, the description of the SKP flows must be done not on line bundles over a supercurve but on line bundles over a family of supercurves parametrized by $\theta, \tau$. To be precise, we are going to consider a family $\mathscr{L}(\theta, \tau)$ of compact complex supermanifolds of dimension $(1,1)$ parametrized by the set of global Grassmann variables $(\theta, \boldsymbol{\tau})$. We will describe this structure in detail. Let $\mathscr{C}=(U ; z, \alpha)$ and $\mathscr{\mathscr { C } ^ { \prime }}=\left(U^{\prime} ; z^{\prime}, \alpha^{\prime}\right)$ be two charts, where the pair $\left\{U, U^{\prime}\right\}$ is a covering of the Riemann sphere, for instance,

$$
U=\{z \in \mathbb{C}|| z \mid<r\}, \neg \quad U^{\prime 0}=\left\{z \in \mathbb{C}|| z \mid>r^{\prime}\right\}, \quad 0<r^{\prime}<r,
$$

with the two systems of coordinates being related through the equations

$$
z^{\prime}=\frac{1}{z}, \quad \alpha^{\prime}=\alpha-\lambda(z, \theta, \tau)
$$

Here $\lambda(z, \theta, \tau)$ is the function defined in (12) and (13) and the transformation $\alpha \mapsto \alpha^{\prime}$ will be called a super-Schiffer transformation. Notice that these supermanifolds are of extended type in the sense of Rogers ${ }^{26}$ because superfunctions on $\mathscr{L}(\theta, \tau)$ depend not only on the local coordinates $(z, \alpha)$ of the supermanifold but also on the global variables $(\theta, \tau)$. In general, the equation (20) for the super-Schiffer transformation $\alpha \mapsto \alpha^{\prime}$ does not extend holomorphically throughout $U^{\prime}$, generically it has a pole of order 1 at $\infty$, and the supermanifold structures defined by the transition superfunctions (20) are not equivalent to the trivial super-Riemann sphere.

Thus we can think on the supermanifold structures defined by the $(\theta, \tau)$-dependent transition functions (20) as tracing a path on the moduli space of complex supercurves. See Ref. 27 for the description of universal deformations on the supermoduli space of supercurves. We could consider further deformations of our complex supercurves but we will simplify the description of superwave functions by considering only the supercurves parametrized by $\theta$ and $\tau$ by means of (20).

We shall consider then the family $\mathscr{L}(x, \mathbf{t})$ of holomorphic line bundles over $\mathscr{L}(\theta, \boldsymbol{\tau})$ with transition function given by the vacuum super-wave function $f_{0}$ of a SKP hierarchy. Our purpose now will be to characterize holomorphic sections of these bundles. Such sections will be determined by pairs

$$
(\mathscr{C} 6, \phi), \quad\left(\mathscr{C}^{\prime}, \phi^{\prime}\right),
$$

defined on the patches $U$ and $U^{\prime}$, and where $\phi$ and $\phi^{\prime}$ are even superfunctions

$$
\begin{gathered}
\phi \in M(U) \otimes \Lambda(\alpha) \otimes C[[x, \mathbf{t}]] \otimes \Lambda(\theta, \boldsymbol{\tau}) \otimes \mathscr{A}, \\
\phi^{\prime} \in M\left(U^{\prime}\right) \otimes \Lambda\left(\alpha^{\prime}\right) \otimes \mathrm{C}[[x, \mathbf{t}]] \otimes \Lambda(\theta, \boldsymbol{\tau}) \otimes \mathscr{C},
\end{gathered}
$$


verifying the overlapping condition,

$$
\left.\left.\phi=\phi^{\prime} \cdot f_{0},\right\urcorner \quad \forall z \in U \cap U^{\prime} \cdot\right\urcorner
$$

Here, $M(U)$ and $M\left(U^{\prime}\right)$ denote the sets of complex valued meromorphic functions of $z$ on $U$ and $U^{\prime}$, respectively.

Comparing our last equation (21) with the factorization property for a super-wave function (17), we immediately see that wide classes of super-wave functions for the SKP hierarchies can be obtained from special sections of $\mathscr{L}(x, \mathbf{t})$. We will search for the conditions defining subsets

$$
\mathscr{W} \subset M(U) \otimes \Lambda(\alpha) \otimes C[[x, \mathbf{t}]] \otimes \Lambda(\theta, \boldsymbol{\tau}) \otimes \mathscr{A},
$$

whose elements will be super-wave functions.

Definition 3: A subspace $\mathscr{W}$ of the the superalgebra $M(U) \otimes \Lambda(\alpha) \otimes C[[x, \mathbf{t}]] \otimes \Lambda(\theta, \boldsymbol{\tau}) \otimes \mathscr{A}$ will be called an asympotic super-module if it verifies:

(C1) $\mathscr{W}$ is a left-module over the algebra of superdifferential operators generated by $D$ and $\left\{\left(\partial / \partial t_{n}\right),\left(\partial / \partial \tau_{n}\right)\right\}_{n \geqslant 1}$ with coefficients in $\mathscr{S}$.

(C2) There is only one even section of the line superbundle $\mathscr{L}(x, \mathbf{t})$ given by the local expressions,

$$
\left.(\mathscr{C}, f),\urcorner\left(\mathscr{C}^{\prime}, f^{\prime}\right),\right\urcorner
$$

such that $f \in \mathscr{W}$ and $f^{\prime}$ is holomorphic on $U^{\prime}$ with $\left.f^{\prime}\right|_{\{z=\infty\}}=1$.

In Section III we will construct explicitly several examples of asymptotic super-modules for complex supercurves with body manifold the Riemann sphere. For more general complex supercurves $\mathscr{L} b$ with body manifold an arbitrary compact Riemann surface $\Sigma$, the previous characterization works similarly. To construct the subspaces $\mathscr{W}$ we should choose first a line bundle $\mathscr{L}$ over $\mathscr{l} b$ such that $\operatorname{dim} H^{0}(\mathscr{L}, \mathscr{L})=\operatorname{dim} H^{1}(\mathscr{l}, \mathscr{B})=0$. Then, fixing a point $p \in \Sigma$ and a local trivialization in a neighborhood of it, we define the new bundle $\mathscr{L} \otimes \mathscr{L}_{p}$ possessing the required properties. Notice that the cohomological requirements on $\mathscr{D}$ implies that $\operatorname{deg} E=0$ where $E$ is the underlying complex line bundle over the Riemann surface. For the Riemann sphere case, this implies that deg $\mathscr{b}=-1$ and the complex supercurve is not a SRS.

We can prove now the following theorem characterizing super-wave functions using asymptotic super-modules.

Theorem 2: Given an asymptotic super-module $\mathscr{W}$, the superfunction

$$
\left.f=f^{\prime} \cdot f_{0},\right\urcorner \quad z \in U \cap U^{\prime},
$$

defined by the unique section associated to it is a super-wave function for the associated SKP hierarchy.

Proof: To see this, observe that from (C2) in Def. 3 we deduce

$$
f^{\prime}=1+\sum_{n=1}^{\infty} w_{2 n}\left(z^{\prime}\right)^{n}+\alpha^{\prime} \cdot \sum_{n=1}^{\infty} w_{2 n-1}\left(z^{\prime}\right)^{n}=1+\sum_{n=1}^{\infty} w_{2 n} z^{-n}+(\alpha-\lambda) \sum_{n=1}^{\infty} w_{2 n-1} z^{-n}
$$

Therefore $f$ satisfies the condition (P2) for super-wave functions. On the other hand, by using (14) we get that for $z \in U \cap U^{\prime}$

$$
\begin{gathered}
D^{2 n} f-w_{1} D^{2 n-1} f=\left[z^{n}+O\left(z^{n-1}\right)\right] f_{0}, \\
D^{2 n+1} f+w_{1} D^{2 n} f=\left[-(\alpha-\lambda) z^{n}+O\left(z^{n-1}\right)\right] f_{0} .
\end{gathered}
$$


As a consequence, we may determine superdifferential operators $P_{n}, Q_{n} \in \mathscr{E}$ such that

$$
\begin{gathered}
\frac{\partial f}{\partial t_{n}}-P_{n} f=O\left(\frac{1}{z}\right) \cdot f_{0}, \\
\frac{D f}{D \tau_{n}}-Q_{n} f=O\left(\frac{1}{z}\right) \cdot f_{0}, \neg n \geqslant 1 .
\end{gathered}
$$

Then, it is easy to see that (C2) implies that both $\partial f / \partial t_{n}-P_{n} f$ and $D f / D \tau_{n}-Q_{n} f, n \geqslant 1$, vanish, and therefore $f$ satisfies the condition (P1) for super-wave functions.

Solutions to a reduced hierarchy (4) can be obtained by imposing on $\mathscr{W}$ the additional condition

$$
z^{r} \cdot \mathscr{W} \subset \mathscr{W} \cdot \neg
$$

Indeed, in that case the super-wave function $f$ associated with $\mathscr{W}$ satisfies

$$
\frac{\partial f}{\partial t_{r}}=z^{r} \cdot f
$$

so that $f^{\prime}$ and $K$ are independent on $t_{r}$.

\section{B. Solitons and solitinos}

We will now construct several examples of asymptotic super-modules and the corresponding super-wave functions.

Let us consider the subset of superfunctions $\phi$ which are analytic for all $z \in U$, with the possible exception of simple poles contained in a fixed subset $\left\{q_{i}\right\}_{i=1}^{N}$, and such that the corresponding residues satisfy

$$
\operatorname{Res}\left(\phi, q_{i}\right)=\left(c_{i}+\alpha \omega_{i}\right) \cdot \phi\left(p_{i}, \alpha\right), \quad i=1, \ldots, N,
$$

where $\left\{c_{i}\right\}_{i=1}^{N} \in \mathscr{b}_{0}$ and $\left\{\omega_{i}\right\}_{i=1}^{N} \in \mathscr{C}_{1}$ are given, and $\left\{p_{i}\right\}_{i=1}^{N}$ is a fixed subset of points of $U$ different from $\left\{q_{i}\right\}_{i=1}^{N^{-}}$. This subspace will be denoted by $\mathscr{W}(\mathbf{q}, \mathbf{p} ; \mathbf{c}, \boldsymbol{\omega})$, where $\mathbf{q}=\left(q_{1}, \ldots, q_{N}\right)$, etc.

Theorem 3: The subspace $\mathscr{W}(\mathbf{q}, \mathbf{p} ; \mathbf{c}, \boldsymbol{\omega})$ is an asymptotic super-module and its elements are super-wave functions for a SKP hierarchy.

Proof: In order to prove that $\mathscr{W}(\mathbf{q}, \mathbf{p} ; \mathbf{c}, \boldsymbol{\omega})$ verifies the required conditions $(\mathrm{C} 1)$ and $(\mathrm{C} 2)$ of Def. 3, let us note that the corresponding superfunction $f$ must be of the form

$$
f=\left[1+\sum_{i=1}^{N} \frac{a_{i}+(\alpha-\lambda) \beta_{i}}{z-q_{i}}\right] \cdot f_{0}, \neg
$$

where the unknown coefficients $a_{i}$ and $\beta_{i}$ are even and odd elements of $\mathscr{S}$, respectively. Thus computing the residues, (27) implies

$$
a_{i}+\left(\alpha-\lambda\left(q_{i}\right)\right) \beta_{i}=\left(b_{i}+\alpha \gamma_{i}\right)\left[1+\sum_{j=1}^{N} \frac{a_{j}+\left(\alpha-\lambda\left(p_{i}\right)\right) \beta_{j}}{p_{i}-q_{j}}\right], \neg
$$

for all $i=1, \ldots, N$, where $b_{i}, \gamma_{i} \in \mathscr{S}$ are given by

$$
b_{i}=e\left(p_{i}\right) c_{i} e\left(q_{i}\right)^{-1}, \quad \gamma_{i}=e\left(p_{i}\right)\left[\omega_{i}+c_{i} \sum_{n \geqslant 1}\left(p_{i}^{n-1}-q_{i}^{n-1}\right) \tau_{n}\right] e\left(q_{i}\right)^{-1}
$$


and with $e(z)$ denoting the superfunction

$$
e(z)=\exp \left[z x+\sum_{n \geqslant 1} z^{n}\left(t_{n}+\theta \tau_{n}\right)-\lambda(z)\left(\theta+\sum_{n \geqslant 1} z^{n-1} \tau_{n}\right)\right] .
$$

From (29) the following linear system for the coefficients $a_{i}$ and $\beta_{i}$ follows

$$
\begin{aligned}
& \sum_{j}\left[\delta_{i j}-\frac{b_{i}}{p_{i}-q_{j}}\right] \cdot a_{j}-\sum_{j}\left[\delta_{i j} \lambda\left(q_{i}\right)-\frac{b_{i} \lambda\left(p_{i}\right)}{p_{i}-q_{j}}\right] \cdot \beta_{j}=b_{i}, \\
& -\sum_{j} \frac{\gamma_{i}}{p_{i}-q_{j}} \cdot a_{j}+\sum_{j}\left[\delta_{i j}+\frac{\gamma_{i} \lambda\left(p_{i}\right)-b_{i}}{p_{i}-q_{j}}\right] \cdot \beta_{j}=\gamma_{i} .
\end{aligned}
$$

This is a uniquely solvable system since the body of the matrix of coefficients is the invertible numerical matrix,

$$
\left(\begin{array}{cc}
\delta_{i j}-\frac{\epsilon\left(b_{i}\right)}{p_{i}-q_{j}} & 0 \\
0 & \delta_{i j}-\frac{\epsilon\left(b_{i}\right)}{p_{i}-q_{j}}
\end{array}\right) \cdot \neg
$$

Therefore we conclude that (28) is a super-wave function.

Theorem 1 allows the identification of the coefficients of the expansion of the super-wave function with the coefficients of the expansion of the operator $K$ solving Sato's equations (2). Observe then for $f$ given by eq. (28), the first two coefficients of the expansion of the associated solution $K$ of Sato equations are

$$
\left.\left.w_{1}=\sum_{i=1}^{N} \beta_{i},\right\urcorner w_{2}=\sum_{i=1}^{N} a_{i}\right\urcorner
$$

We consider the following two different cases for the system (30).

\section{Supersoliton solutions: $\epsilon\left(c_{i}\right)=0, \forall i \neq 1, \ldots, N$}

From (30) it is easy to see that the body of $f$ is the wave function associated with the $N$-soliton solution of the KP hierarchy. Therefore the solution of the SKP hierarchy provided by $f$ is a supersymmetric generalization of the standard multisoliton solutions. For example, it is rather simple to derive the explicit solution for $N=1$. In this case, the constraints (27) reduce to

$$
\operatorname{Res}(\phi, q)=(c+\alpha \omega) \phi(p, \alpha) .
$$

Then one finds

$$
f=\left[1+\frac{a+(\alpha-\lambda) \beta}{z-q}\right] \cdot f_{0},
$$

with

$$
\begin{gathered}
a=\frac{(q-p) b}{q-p+b}+\frac{\lambda(p) b+(q-p) \lambda(q)}{(q-p+b)^{3}}(q-p)^{2} \gamma, \\
\beta=\frac{(p-q)^{2}}{(q-p+b)^{2}} \gamma .
\end{gathered}
$$






FIG. 1. Composite structure of a single supersoliton, $\left(h_{1}(x, t), q=1, x_{0}=1\right)$.

If we take $p=-q$, then it follows that $z^{2} \cdot \mathscr{W} \subset \mathscr{W}$, so that $f$ becomes a super-wave function for the supersymmetric KdV hierarchy. In particular, it provides a solution of the SKdV equation (8). To simplify the expression of this solution we set $t_{n}=0, \forall n \neq 3$, and $\tau_{n}=0, \forall n \geqslant 1$. Thus, from (7), (32) and (33), we find

$$
\begin{gathered}
v_{0}=2\left(q^{2}+\theta \omega \frac{q^{3}}{c}\right) \operatorname{sech}^{2}\left[q\left(x-x_{0}+q^{2} t\right)\right]-3 \theta \omega \frac{q^{3}}{c} \operatorname{sech}^{4}\left[q\left(x-x_{0}+q^{2} t\right)\right], \\
v_{1}=\omega \frac{2 q^{2}}{c} \sinh \left[q\left(x-x_{0}+q^{2} t\right)\right] \operatorname{sech}^{3}\left[q\left(x-x_{0}+q^{2} t\right)\right],
\end{gathered}
$$

where

$$
x_{0}=\frac{1}{q} \log \left(\frac{c}{2 q}\right)
$$

The expression of $v_{0}$ may be described as a supersymmetric dressing of the standard soliton of the KdV equation. Writing $v_{0}=h_{0}(x, t)+h_{1}(x, t) \theta \omega$ and $v_{1}=h_{2}(x, t) \omega$, in Fig. 1 we show the composite structure of $h_{1}$, the $\theta \omega$ component of the superfunction $v_{0}$.

On the other hand, it is worth noticing that the odd superfunction $v_{1}$ is proportional to a function $h_{2}$ of $(x, t)$ which represents also a composite structure (see Fig. 2) which propagates without deformation along the $x$ axis.

\section{Solitino solutions: $c_{i}=0, \forall i=1, \ldots, N$}

In this case (29) and (30) reduces to

$$
\begin{gathered}
a_{i}=\lambda\left(q_{i}\right) \cdot \beta_{i}, \\
\gamma_{i}=\sum_{j=1}^{N}\left[\delta_{i j}+\gamma_{i} \frac{\lambda\left(p_{i}\right)-\lambda\left(q_{j}\right)}{p_{i}-q_{j}}\right] \beta_{j} .
\end{gathered}
$$

This system can be solved in terms of the nilpotent matrix

$$
\Delta_{i j}=\frac{\lambda\left(p_{i}\right)-\lambda\left(q_{j}\right)}{p_{i}-q_{j}} \gamma_{i} . \neg
$$

Indeed, as $\Delta^{N} \boldsymbol{\gamma}=0$ the solution of (35) is 




FIG. 2. Odd component of a supersoliton $\left(h_{2}(x, t), q=1, x_{0}=1\right)$.

$$
\left.\boldsymbol{\beta}=\sum_{n=0}^{N-1} \Delta^{n} \boldsymbol{\gamma}\right\urcorner
$$

where $\boldsymbol{\beta}=\left(\beta_{1}, \ldots, \beta_{N}\right)$ and $\boldsymbol{\gamma}=\left(\gamma_{1}, \ldots, \gamma_{N}\right)$. It must be observed that the body of $f$ vanishes, so that this class of solutions is not related to any class of the standard KP theory. We will refer to these solutions as solitino solutions. The single solitino solution corresponds to $N=1$ and takes the form

$$
\begin{gathered}
f=\left[1+\frac{a+(\alpha-\lambda) \beta}{z-q}\right] \cdot f_{0}, \\
w_{1}=\beta=\omega \exp r, \quad w_{2}=a=\lambda(q) \omega \exp r,
\end{gathered}
$$

where

$$
\begin{aligned}
\exp r= & e(p) e(q)^{-1}=\exp \left[(p-q) x+\sum_{n \geqslant 1}\left(p^{n}-q^{n}\right)\left(t_{n}+\theta \tau_{n}\right)-(\lambda(p)-\lambda(q)) \theta\right. \\
& \left.\left.-\sum_{n \geqslant 1}\left(\lambda(p) p^{n-1}-\lambda(q) q^{n-1}\right) \tau_{n}\right] .\right\urcorner
\end{aligned}
$$

If we take $p_{i}=-q_{i}, \forall i=1, \ldots, N$, then $z^{2} \cdot \mathscr{W} \subset \mathscr{W}$ and we obtain a solution of (8). It adopts a convenient way by setting, as we did before, $t_{n}=0, \forall n \neq 3$ and $\tau_{n}=0, \forall n \geqslant 1$. Thus the matrix (36) reduces to

$$
\Delta_{i j}=\theta \omega_{i} \exp \left[-2 q_{i}\left(x+q_{i}^{2} t\right)\right]
$$

and

$$
\gamma_{i}=\omega_{i} \exp \left[2 q_{i}\left(x+q_{i}^{2} t\right)\right],
$$






FIG. 3. An even component of a bisolitino solution.

and we find the following multisolitino solution of the SKdV equation (8):

$$
\begin{gathered}
v_{0}=4 \theta \sum_{i} q_{i}^{2} \omega_{i} \exp \left[-2 q_{i}\left(x+q_{i}^{2} t\right)\right]+4 \sum_{i, j} q_{i} \omega_{i} \omega_{j} \exp \left[-2 q_{i}\left(x+q_{i}^{2} t\right)-2 q_{j}\left(x+q_{j}^{2} t\right)\right], \\
\left.v_{1}=4 \sum_{i} q_{i} \omega_{i} \exp \left[-2 q_{i}\left(x+q_{i}^{2} t\right)\right] .\right\urcorner
\end{gathered}
$$

In particular the single solitino solution takes the form

$$
\left.v_{0}=4 q^{2} \theta \omega \exp \left[-2 q\left(x+q^{2} t\right)\right],\right\urcorner v_{1}=4 q \omega \exp \left[-2 q\left(x+q^{2} t\right)\right] .
$$

It is not a localized solution as a function of $x$. In fact, for imaginary $q$ it is a plane wave.

The multisolitino solution (39) represents a superposition of solitinos which is of a nonlinear character, as it is shown by the presence of quadratic terms in the expression for $v_{0}$. See Fig. 3 for a representation of a component of the even part of a bisolitino solution.

\section{Rational solutions}

A solution $K$ of the SKP hierarchy (2) is said to be rational if the coefficients $w_{n}$ of its expansion (3) are rational functions of the even coordinates $(x, \mathbf{t})$. Solutions of this kind can be derived by means of asymptotic super-modules similar to those used for solitons and solitinos by imposing instead of the conditions (27) constraints of the type

$$
\left(\alpha \frac{\partial}{\partial \alpha} \frac{\partial^{n_{i}}}{\partial z^{n_{i}}}+c_{i} \frac{\partial^{m_{i}}}{\partial z^{m_{i}}}\right) \phi\left(p_{i}, \alpha\right)=0 .
$$

For example, let us take $\mathscr{W}$ to be the set of superfunctions $\phi$ analytic on $U$ with the possible exception of a simple pole at a given point $q \neq 0$, and such that

$$
\alpha \frac{\partial}{\partial \alpha} \frac{\partial \phi}{\partial z}(0, \alpha)+\phi(0, \alpha)=0 .
$$


It is straightforward to get

$$
\left.f=\left[1+\frac{a+(\alpha-\lambda) \beta}{z-q}\right] \cdot f_{0},\right\urcorner
$$

with

$$
a=q, \quad \beta=-\frac{q\left(\theta+\tau_{1}\right)}{1+q\left(x+t_{1}+1\right)} .
$$

As $z^{2} \cdot \mathscr{W} \subset \mathscr{W}$ this construction leads to a solution of (8) which turns out to be given by

$$
\left.v_{0}=0,\right\urcorner \quad v_{1}=-\frac{2 q^{2}\left(\theta+\tau_{1}\right)}{\left[1+q\left(x+t_{1}+1\right)\right]^{2}} .
$$

Another simple example is obtained by replacing (41) by

$$
\frac{\partial \phi}{\partial z}(0, \alpha)=0,
$$

then the superfunctions $a$ and $\beta$ in (42) take the form

$$
a=q\left(1-\frac{1}{s}+\frac{r \tau_{2}}{s^{3}}\right), \quad \beta=\frac{q^{2} \tau_{2}}{s^{2}},
$$

where for the MRKP hierarchy

$$
\left.s=q\left(x+t_{1}+\tau_{1} \theta\right)+1,\right\urcorner r=q^{2}\left(\theta-\tau_{1}\right),
$$

and for the JKP hierarchy

$$
\left.s=q\left(x+t_{1}\right)+1,\right\urcorner \quad r=q^{2} \theta .
$$

The condition $z^{2} \cdot \mathscr{W} \subset \mathscr{W}$ keeps on being satisfied and the corresponding solution of (8) is

$$
v_{0}=-\frac{2 q^{2}}{s^{2}}+\frac{6 q^{2} r \tau_{2}}{s^{4}}, \neg v_{1}=\frac{4 q^{3} \tau_{2}}{s^{3}} .
$$

We notice that this solution is a supersymmetric dressing of the elementary rational solution

$$
v=\frac{-2 q^{2}}{(q x+1)^{2}}
$$

of the KdV equation.

\section{SUPERGROUP THEORETICAL DESCRIPTION}

It is well known the use of $\bar{\partial}$-equations to obtain wave functions for the KP hierarchy. ${ }^{21,22}$ This theory was extended to the supersymmetric situation in Ref. 23.

We shall consider solutions of the $\bar{\partial}$-equation,

$$
\frac{\partial}{\partial \bar{z}} f(z, \alpha ; x, \theta ; \mathbf{t}, \boldsymbol{\tau})=\int d^{2} z^{\prime} d \alpha^{\prime} a\left(z^{\prime}, \alpha^{\prime} ; z, \alpha\right) f\left(z^{\prime}, \alpha^{\prime} ; x, \theta ; \mathbf{t}, \boldsymbol{\tau}\right),
$$


with $a\left(z^{\prime}, \alpha^{\prime} ; z, \alpha\right)$ a given distribution of odd parity. We shall denote by $\mathscr{F}$ the space of solutions of Eq. (44) having an asymptotic expansion, $z \rightarrow \infty$, of the form

$$
F=\left(\sum_{n=-\infty}^{N} u_{n} z^{n}+(\alpha-\lambda) \sum_{n=-\infty}^{M} v_{n} z^{n}\right) f_{0},
$$

with coefficients $u_{n}, v_{n}$ in $\mathscr{S}$.

By using the properties (9) of the vacuum super-wave function $f_{0}$ and the isospectrality of the $\bar{\partial}$-equation (44) (the kernel $a$ of the equation (44) does not depend on $x, t$ ), it is immediate that $\mathscr{F}$ is a left module for the algebra of superdifferential operators $\mathscr{E}$. Then, if a unique even solution $f$ of Eq. (44) with the asymptotic expansion Eq. (18) exists, then by Thm. 2, it will be a superwave equation for a SKP hierarchy.

It was shown in Ref. 23 that solutions of the $\bar{\partial}$-equation are related with the $\tau$-function of the SKP hierarchy generated from the action of the element

$$
g=\exp \frac{1}{\pi} \int d^{2} z^{\prime} d \alpha^{\prime} d^{2} z d \alpha B\left(z^{\prime}, \alpha^{\prime}\right) a\left(z^{\prime}, \alpha^{\prime} ; z, \alpha\right) C(z, \alpha)
$$

of the supergroup $G L(\infty \mid \infty)$ on the vacuum. The quantum fields $B, C$ form a superghost system with commutation and anticommutation relations given by

$$
\left[B(z, \alpha), B\left(z^{\prime}, \alpha^{\prime}\right)\right]=\left\{C(z, \alpha), C\left(z^{\prime}, \alpha^{\prime}\right)\right\}=0 ; \quad\left[B(z, \alpha), C\left(z^{\prime}, \alpha^{\prime}\right)\right]=\left(\alpha-\alpha^{\prime}\right) \hat{\delta}_{\gamma}\left(z-z^{\prime}\right),
$$

and $\hat{\delta}_{\gamma}(z)$ denotes the delta function with support on the circle $\gamma$ acting on test functions as

$$
\int d^{2} z \hat{\delta}_{\gamma}(z) \phi(z)=\oint_{\gamma} z \phi(z)
$$

One of the advantages of the $\tau$-function formalism for integrable systems is that it allows us to classify the solutions in terms of orbits of symmetry groups. For the SKP hierarchies considered in this paper a $\tau$-function description is already available which exhibits their invariance under the supergroup $G L(\infty \mid \infty){ }^{23} \mathrm{We}$ can thus conclude form the above discussion that the $\tau$-function of the SKP hierarchy generated from the action of (45) on the vacuum is associated with the superwave function which satisfies the corresponding $\bar{\partial}$ equation.

As for the solutions analyzed in this paper, it is easy to derive the $\bar{\partial}$-equation verified by their associated super-wave functions. For example, the solutions that arise from the constraints (27) and (41) give rise to the kernels

$$
a\left(z^{\prime}, \alpha^{\prime} ; z, \alpha\right)=\pi \sum_{i=1}^{N}\left(\alpha^{\prime}-\alpha\right)\left(c_{i}+\alpha^{\prime} \omega_{i}\right) \delta\left(z-q_{i}\right) \delta\left(z^{\prime}-p_{i}\right)
$$

and

$$
\left.a\left(z^{\prime}, \alpha^{\prime} ; z, \alpha\right)=\frac{\delta(z-q)}{2 \pi i}\left[\pi\left(\alpha^{\prime}-\alpha\right) \hat{\delta}_{\gamma_{1}}\left(z^{\prime}\right)+\left(\frac{\alpha^{\prime}-\alpha}{z^{\prime}}-\frac{\alpha}{\left(z^{\prime}\right)^{2}}\right) \hat{\delta}_{\gamma_{2}}\left(z^{\prime}\right)\right],\right\urcorner
$$

respectively. Here $\delta(z-q)$ is the usual delta function on the plane and $\hat{\delta}_{\gamma_{i}}(z), i=1,2$, denotes the delta functions with support on the circles $\gamma_{i}\left(|z|=r_{i}\right)$, with $r_{1}>|q|>r_{2}$. Consequently, we have provided two additional different characterizations for our solutions of the SKP hierarchies. First, from Eq. (45) every solution is associated with an element of $G L(\infty \mid \infty)$. Second, Eq. (44) shows that the kernel $a$ supplies the spectral description of the solutions. 


\section{ACKNOWLEDGMENTS}

The authors would like to thank the financial support of CICYT under project PB92-0197.

${ }^{1}$ M. R. Douglas, Phys. Lett. B 238, 176 (1990).

${ }^{2}$ R. Dijkgraff, H. Verlinde, and E. Verlinde, Nucl. Phys. B 348, 435 (1991).

${ }^{3}$ V. E. Zakharov, S. V. Manakov, S. P. Novikov, and L. P. Pitaevskii, Theory of Solitons: the Inverse Scattering Method (Plenum, New York, 1984).

${ }^{4}$ E. Date, M. Jimbo, M. Kashiwara, and T. Miwa, “Transformation groups for soliton equations”, in Nonlinear Integrable Systems-Classical and Quantum Theory, edited by M. Jimbo and T. Miwa (World Scientific, Singapore, 1983).

${ }^{5}$ M. Sato and Y. Sato, US-Japan Seminar, Nonlinear Partial Differential Equations in Applied Sciences, edited by H. Fujita, P. D. Lax, and G. Strang (Kinokuniya/North-Holland, Amsterdam, 1982), p. 259.

${ }^{6}$ M. F. Atiyah, The Geometry and Dynamics of Magnetic Monopoles (Princeton U. P., Princeton, NJ, 1988).

${ }^{7}$ G. Segal and G. Wilson, Publ. Math. IHES 61, 5 (1985).

${ }^{8}$ J. Palmer, Acta Appl. Math. 18, 199 (1990).

${ }^{9}$ K. Ueno and H. Yamada, Adv. Stud. Pure Math. 16, 373 (1988).

${ }^{10}$ M. Mulase, J. Diff. Geom. 34, 651 (1991).

${ }^{11}$ J. M. Rabin, Commun. Math. Phys. 137, 533 (1991).

${ }^{12}$ L. Alvarez-Gaumé, H. Itoyama, J. L. Mañes, and A. Zadra, Int. J. Mod. Phys. A 7, 5337 (1992).

${ }^{13}$ L. Alvarez-Gaumé, K. Becker, M. Becker, R. Emperan, and J. Mañes, Int. J .Mod. Phys. A 8, 2297 (1993).

${ }^{14}$ K. Becker and M. Becker, Mod. Phys. Lett. A 8, 1205 (1993).

${ }^{15}$ Yu I. Manin and A. O. Radul, Commun. Math. Phys. 98, 65 (1985).

${ }^{16}$ W. Oevel and Z. Popowicz, Commun. Math. Phys. 139, 41 (1990).

${ }^{17}$ I. N. McArthur, Commun. Math. Phys. 139, 121 (1994).

${ }^{18}$ P. Mathieu, Phys. Lett. B 203, 287 (1988).

${ }^{19}$ A. Bilal and J. Gervais, Phys. Lett. B 211, 95 (1988).

${ }^{20}$ M. Mañas, L. Martinez Alonso, and E. Medina, Phys. Lett. B 336, 178 (1994).

${ }^{21}$ R. Beals and R. Coifman, Physica D 18, 242 (1986).

${ }^{22}$ M. Jaulent, M. Manna, and L. Martinez Alonso, Inv. Prob., 4, 123 (1988).

${ }^{23}$ L. Martinez Alonso and E. Medina, J. Math. Phys. 36, 4898 (1995).

${ }^{24}$ K. Ueno, H. Yamada, and K. Ikeda, Commun. Math. Phys. 124, 57 (1989).

${ }^{25}$ Y. Manin, Gauge field theory and complex geometry, Grundlehren Math. Wiss., Vol. 289 (Springer-Verlag, New York, 1988).

${ }^{26}$ A. Rogers, Commun. Math. Phys. 105, 375 (1986).

${ }^{27}$ G. Falqui and C. Reina, Commun. Math. Phys. 128, 247 (1990). 
Journal of Mathematical Physics is copyrighted by AIP Publishing LLC (AIP). Reuse of AIP content is subject to the terms at: http://scitation.aip.org/termsconditions. For more information, see http://publishing.aip.org/authors/rights-and-permissions. 